\title{
3D Face Reconstruction from a Single Shaded Image Using Subspace Crossing Engine
}

\author{
Tanasai Sucontphunt \\ Graduate School of Applied Statistics \\ National Institute of Development Administration \\ Thailand
}

\begin{abstract}
This paper presents a technique to reconstruct a 3D human face from a single shaded face image. The core idea of this work is to find a possible relationship between an image subspace and a 3D subspace. In this work, the subspaces are created by Principle Component Analysis to only capture the major factors for creating a face in each subspace. The subspaces are then adjusted to arrange them in a comparable range. By inputting a novel $2 \mathrm{D}$ shading face image, a subspace crossing engine developed for this work will estimate the major factors of the $2 \mathrm{D}$ shading face image creation and use them to compose a 3D shape automatically. The evaluation reveals that the researchers approach reconstructed 3D faces faster and closer to the 3D ground-truth faces than a traditional approach in a controlled environment.
\end{abstract}

Keywords: Shape from Shading, 3D Face Reconstruction, 3D Face Modeling.

\section{Introduction}

The Shape-from-Shading (SfS) [1] is a challenge problem in computer vision and graphics. It only tries to understand a 3D shape from a single image; a shaded image of the 3D shape. Typically, it can be achieved by estimating parameters in a rendering equation to match the shaded image. However, there is a huge variation of the parameters in creating the same pixel values in an image. Thus, a data-driven approach is typically used to only limit the range of the parameters to the possible values. This datadriven approach learns the pattern of shapes from a collection of existing 3D shapes. The approach is particularly useful for reconstructing a 3D shape with a small variation such as a human face.

From an input face image (in a frontal view, typically), a 3D face model can be reconstructed by a morphable face model. The morphable model is constructed from a collection of 3D shapes [2] or directly from 2D images [3]. If an input face image is a photograph, the morphing is processed in 2 subspaces separately: a 3D subspace for 3D shape, and a texture subspace for facial texture image. A 3D face can be modeled by ad justing morphing parameters in 3D subspace as well as in texture subspace to match the face image. Because the morphing parameters of 3D shape are limited to 3D subspace, reconstructing a 3D face from a 2D image is achieved by Analysis-by-Synthesis (AbS) approach. Using the AbS approach, it iteratively compares and analyzes an input 2D image with its synthesized 2D image. The synthesized 2D image is created by adjusting 
morphing parameters in 3D subspace as well as texture subspace so that when the face is rendered with a rendering equation, such as Lambertian reflection model, it will match the input 2D image with most associated results. As the synthesis process focuses on both shape and texture at the same time, the resultant 3D model tends to skip minor shape surface details, which appear on the input 2D image such as ridges and valleys. Additionally, this iterative analysis consumes high computational time. The researchers work demonstrates that the hidden semantics between 2D and 3D subspaces are directly exploited in order to compose the 3D shape and its surface in detail. Since a facial texture does not have a direct relationship to its 3D shape, this study focuses entirely on a greyscale shaded image rather than a realistic photography as a proof of our concept.

\section{Related Work}

In addition to the Shape-from-Shading (SfS), simple 3D object shapes can be constructed from the simple images with an inverse process of the Non-Photorealistic Rendering (NPR) [45]. Bourguignon et al. [4] introduced a method to create a 3D object by interpreting its depth cues from a 2D drawing. Gingold et al. [5] developed an intuitive user interface to collect shapes and annotations over a sketch image to produce a 3D object. These approaches demonstrated their successes in crafting simple 3D shapes from lines. However, without a domain-specific knowledge, it is difficult to produce a complex model.

Regarding a 3D face modeling, famous research direction is aiming in utilizing the patterns of the human face to create a realistic face model. Decarlo et al. [6] developed a data-driven technique to generate 3D face models by imposing facial anthropometry statistics as constraints. However, the 3D faces generated by their approach are not sufficiently realistic, since the used anthropometric constraints are too sparse. Notably, Blanz and Vetter [2] built a 3D morphable face model by constructing Principal Component Analysis (PCA) spaces from the 3D geometry and texture of a scanned 3D face dataset to synthesize a 3D face model from a single photograph, a vague information [7], or a facial landmark [8]. Kemelmacher and Basri [9] used a reference 3D model to morph it to depths and normals guided by the input 2D image. Recently, Kemelmacher-Shlizerman [3] used morphable model constructed directly from 2D photos on the internet to model 3D face with facial expression. Their works performed well in creating a human face from a 2D image. Nevertheless, these data-driven approaches are used to craft an overall facial shape. The details of a facial shape, such as ridges and valleys, can only be synthesized with an additional optimization over a rendering equation. New assumptions require modification on an optimization algorithm. In contrast, the researchers work synthesizes the facial details directly from a collection of data using a relationship between different representations of the data. While their approaches normally take high computation and need minutes to complete the task, the researchers work simplifies the prior knowledge to run in linear time, which takes less than a second in rendering. 


\section{Data Preparation}

The data-driven approach is the main concept to deal with the 3D shape reconstruction in this work. Thus, a collection of 3D human faces is first processed in an offline manner to construct proper subspaces to be used in the real-time application. This work is engined by a pre-collected 3D human face dataset consisting of 100 subjects with diversified genders, ages, and ethnicities. The researcher has created three subspaces for 3D face shape, 2D shading image, and 3D rotation matrix as follows.

3D Shape Subspace $\left(\phi_{3 D}\right)$ : The geometry of each 3D face is encoded as vertex positions of the model. Then, the PCA representations of the geometry are constructed using a Singular Value Decomposition (SVD) from the covariance matrix of feature vectors formed by these vertex positions. Each feature vector is constructed by putting all vertices into a column vector as shown in each column of Eq. 1 where $V$ represents the vertex positions, $n$ is the total number of vertices, $f$ is the total number of human faces in the dataset, and $\operatorname{Cov}\left(G_{3 D}\right)$ is a covariance matrix. After SVD, the $\phi_{3 D}$ is the eigenvectors (subspace) with the size of $(3 n) \times(f-1)$.

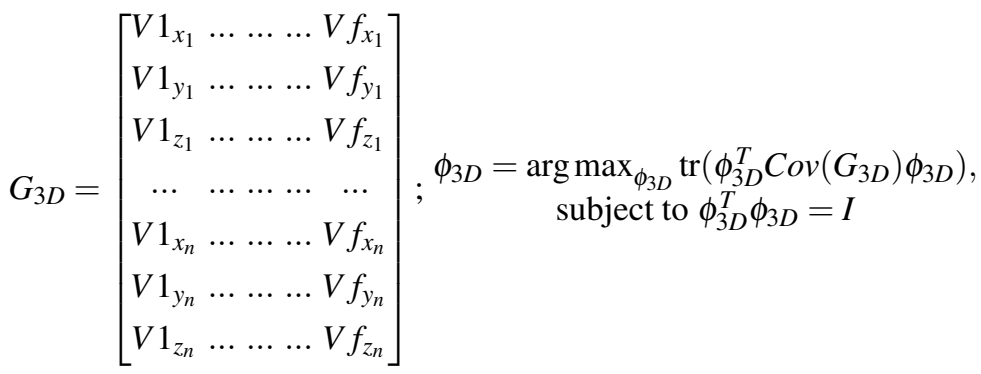

2D Shading Subspace $\left(\phi_{2 D}\right)$ : To interpret the input 2D shading image, in this work, all 3D human faces in the dataset are also rendered by Lambert shading from their frontal view to create 2D shading images of 500x500 square pixels for the faces. Then, the PCA subspace for these 2D images are constructed the same way as the $3 \mathrm{D}$ facial subspace (similar to Eq. 11). A feature vector in the constructing matrix is created from a greyscale value of each $2 \mathrm{D}$ image pixel.

3D Rotation Subspace $\left(\phi_{\log (R)}\right)$ : To craft minor details of a face shape, the direct relation- ship to its deformation operator needs to be constructed. The operator is used to deform the shape only slightly for the minor details. In this work, the operator is encoded as 3D affine transformation matrices between the face and an average human face. To calculate the affine transformations for each face mesh, the researcher employed the deformation gradient algorithm [10]. However, if the affine transformations are directly used to apply to a shape, even a small change in shading information, it will change the whole face shape. This is because the scaling component in the affine transformations does not have a direct relationship to the shading information. In contrast, the rotation component contains a direct relationship to its shading information, for example, a normal direction in a rendering equation. Thus, the researcher decomposed an affine transformation matrix to a rotation component, $R$, and a scaling component, $S$, using polar decomposition. Then, we use the exponential map to transform R to its 
logarithm space, $\log (R)$, to ensure its linear interpolable property of rotation [11]. Concerning each face mesh, we concatenate the $\log (R)$ of all the vertices to form a feature vector. Based on all the feature vectors, we has finally constructed a truncated rotation PCA subspace (similar to Eq.11), namely, $\phi_{\log (R)}$.

2D Shading Gradient Subspace $\left(\phi_{2 D_{\text {Grad }}}\right)$ : To semantically make a connection to the $3 \mathrm{D}$ rotation subspace $\left(\phi_{\log (R)}\right)$, the operator in 2D shading image also needs to be constructed first. This operator is created from an image gradient between a Lambert shading image (as in the 2D shading subspace) of each face in the dataset and an average human face. Each 2D shading gradient consists of 500x500 square pixels. Then, the PCA subspace for these $2 \mathrm{D}$ gradients, $\phi_{2 D_{G r a d}}$, are constructed the same way as the $2 \mathrm{D}$ shading subspace.

\section{System Overview}

The concept of this work is to decompose a shading image into feasible pieces, and then to recompose them to a 3D shape. This process can be achieved by utilizing different, related subspaces which are created from different representations of a face collection, i.e., 2D shading image $\left(\phi_{2 D}\right)$ and 3D shape $\left(\phi_{3 D}\right)$. To do so, first, an input shading image is projected into the $\phi_{2 D}$ to create its semantic in this subspace. Then, a subspace crossing engine (Section 5) developed for this work will transform this semantic to the $\phi_{3 D}$ in order to synthesize a 3D shape. However, the initial 3D shape created from this process still lacks of details, since the $\phi_{3 D}$ can only capture overall face shapes (Figure 2, third row). The details can be added by modifying the initial 3D shape's normals with rotation matrices similar to a bump mapping technique. Nevertheless, unlike a bump mapping technique, our technique actually modifies the orientation of each triangle permanently.

To infer proper rotation matrices, the image gradient between the initial 3D shaped shading image and the input 2D shading image is used to find its corresponding rotation matrices. The analogy between these subspaces is the difference between two images can be used to infer the difference between two surfaces. To skip the rendering process, a reconstructed shading image from the $\phi_{2 D}$ projection is directly used instead (i.e., Reconstructed2DShading $=2$ DShading $* \phi_{2 D} * \phi_{2 D}^{T}$ ). This difference (a.k.a. Residue = 2 DShading - Reconstructed 2 DShading) is then used to project to the $\phi_{2 D_{\text {Grad }}}$ to create its semantic is this subspace. Next, this semantic will be transformed to the $\phi_{\log (R)}$ in order to reconstruct the rotation matrices. These rotation matrices are then used in the deformation gradient framework to deform the orientation of each triangle to its plausible orientation by a least square fitting. Figure 1 illustrates this whole process.

\section{Subspace Crossing Engine}

The core idea of this work is to utilize the relationships between different subspaces. Each subspace is a representation of the same objects in deferent representations (i.e., 2D shading images, 3D vertex positions, 2D gradients and 3D gradients). The main assumption of these relationship creations is that a pattern in creating an object in one 


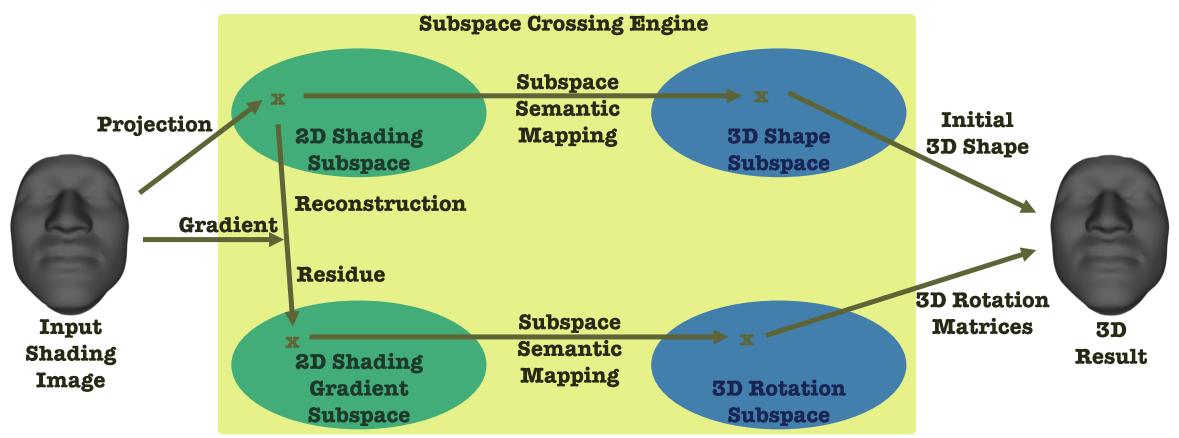

Fig. 1. The overview of our system. Our subspace crossing engine utilizes four relative subspaces to reconstruct a $3 \mathrm{D}$ face model from an input $2 \mathrm{D}$ shading image.

subspace must be similar to another subspace. To be more specific, the PCA coefficients in one subspace are modified to be used to reconstruct an object in an another subspace. Since we carefully select the related representations to cross their subspaces, different representations of the same object still hold many common features.

To construct a 3D shape from a 2D shading subspace, the simplest solution is to manually create a mapping between each eigenvector of the $\phi_{2 D}$ and its actual corresponding $3 \mathrm{D}$ shapes. Then, the PCA coefficients from the $\phi_{2 D}$ can be modified to be used as a weighted sum of these 3D shapes in order to reconstruct a 3D shape. However, the corresponding 3D shapes need to be synthesized individually (if it is even possible) which are against PCA interpolation premises. Firstly, to interpolate properly, these synthesized 3D shapes must be aligned orthogonally in its PCA subspace. Secondly, adjusting PCA coefficients for a weighted sum is not straightforward because these synthesized $3 \mathrm{D}$ shapes have no relation to each other. From this reason, the $\phi_{3 D}$ constructed from the same observations as in the $\phi_{2 D}$ is used instead. Nonetheless, this problem is still ill-posed, because it violates 3 main assumptions of PCA subspace manipulation when the observations (training data) are drawn from different sources (the same objects in different representations) which are:

1. The observations in each representation have different means and variances.

2. The observations in each representation are drawn from different distributions.

3. The observations in each representation are arranged differently in its subspace.

To ameliorate these violations, the subspace configuration framework is introduced to the subspace crossing engine. This framework consists of 3 main steps, including observation normalization, transfer subspace learning, and subspace semantic mapping.

\subsection{Observation Normalization}

The trick of making different observations in each representation comparable is to normalize them first. Otherwise, different subspaces will define their own ranges leading to completely different mean and variance among subspaces. In this work, all observations are normalized by applying z-scores similar to an image normalization technique before SVD process. This will make all representation observations fixed in a comparable range regardless of their dimensions. 


\subsection{Transfer Subspace Learning}

In adjusting the PCA subspace of each representation to share similar distributions, the Transfer Subspace Learning (TSL) is employed in the PCA subspace construction process (Eq.11). Bregman divergence-based regularization [12] is used for TSL in this work, due to its accuracy and simplicity. This regularization aims to adjust PCA subspaces so that their distribution difference is minimized. This can be achieved by using a gradient descent algorithm over the distribution difference measured by Bregman divergence as shown in Eq. 2. Thus, in this work, the $\phi_{2 D}$ is adjusted to have a similar distribution to the $\phi_{3 D}$ and the $\phi_{2 D_{G r a d}}$ is adjusted to have a similar distribution to the $\phi_{\log (R)}$.

$$
\begin{gathered}
\phi_{2 D}=\arg \min _{\phi_{2 D}} \operatorname{tr}\left(\phi_{2 D}^{T} \operatorname{Cov}\left(G_{2 D}\right) \phi_{2 D}\right)+\lambda B\left(D_{2 D} \| D_{3 D}\right), \\
\text { and } \\
\phi_{2 D_{\text {Grad }}}=\arg \min _{\phi_{2 D_{\text {Grad }}}} \operatorname{tr}\left(\phi_{2 D_{\text {Grad }}}^{T} \operatorname{Cov}\left(2 D_{\text {Grad }}\right) \phi_{2 D_{\text {Grad }}}\right)+\lambda B\left(D_{2 D_{\text {Grad }}} \| D_{\log (R)}\right),
\end{gathered}
$$

As shown in the Eq. 2, $B$ is the Bregman divergence measurement, $D_{\text {representation }}$ is a distribution of the observations in the specific representation estimated by kernel density estimation technique, and $\lambda$ is a trade-off parameter between the two terms.

\subsection{Subspace Semantic Mapping}

From the previous two steps, the observations as well as PCA subspace distributions are now in the comparable unit. However, a PCA subspace of each representation may contain different semantics. For example, the first eigenvector of the $\phi_{2 D}$ may refer to facial outline variation, while the first eigenvector of the $\phi_{3 D}$ may refer to mouth shape variation. To mitigate this problem, the mapping between subspaces which links the same semantics between eigenvector from one space to the another is constructed. To do so, each observation in the dataset is projected to the $\phi_{2 D}$ and the $\phi_{3 D}$ according to its representations. The subspace semantic map is then constructed by Eq. 3, where $\operatorname{Proj}_{2 D}$ is a row vector matrix of projected 2D observations, $\operatorname{Proj}_{3 D}$ is a row vector matrix of projected 3D observations, and $M_{2 D \rightarrow 3 D}$ is the subspace semantic map. Similarly, $M_{2 D_{\text {Grad }} \rightarrow \log (R)}$ can also be constructed using $\operatorname{Proj}_{2 D_{\text {Grad }}}$ and $\operatorname{Proj}_{\log (R)}$ instead of $\operatorname{Proj}_{2 D}$ and $\operatorname{Proj}_{3 D}$ respectively.

$$
\begin{aligned}
& M_{2 D \rightarrow 3 D} * \operatorname{Proj}_{2 D}=\operatorname{Proj}_{3 D} \\
& M_{2 D \rightarrow 3 D}=\operatorname{Proj}_{3 D} * \operatorname{Proj}_{2 D}^{-1}
\end{aligned}
$$

These matrices are then used as the main engine to cross from one subspace to another subspace as in the following:

- For 2D shading to 3D shape subspace crossing, 3DShape $=M_{2 D \rightarrow 3 D} * 2 \mathrm{DSh}$ ading.

- For 2D shading gradient to 3D rotation subspace crossing, 3 DRotation $=M_{2 D_{\text {Grad }} \rightarrow \log (R)} * 2$ DShadingGradient. 


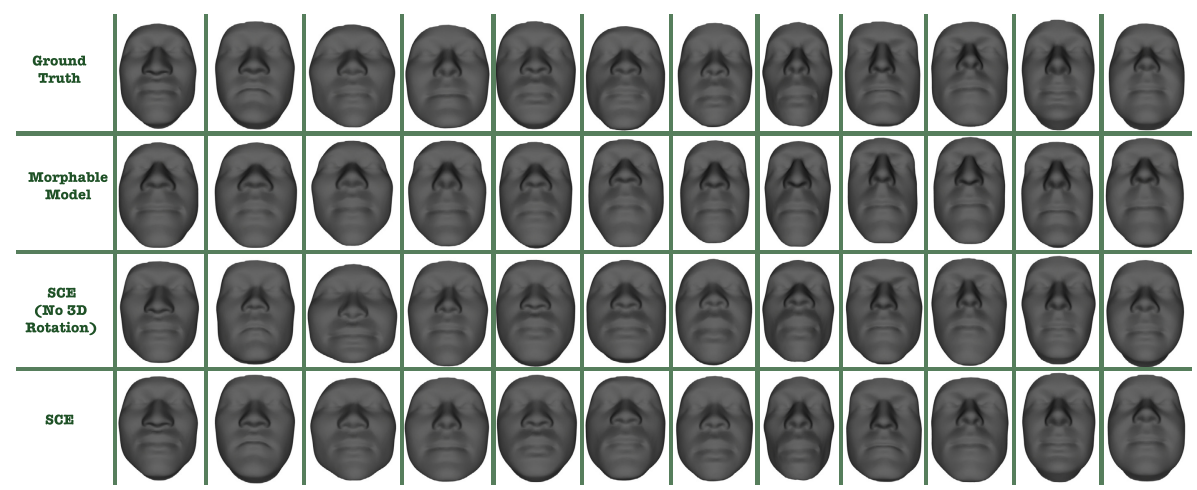

Fig. 2. Visually comparing (in each column) the reconstructed results generated from a 3D morphable model technique, our SCE without using 3D rotation matrices, and our main SCE technique to the ground truth shading images

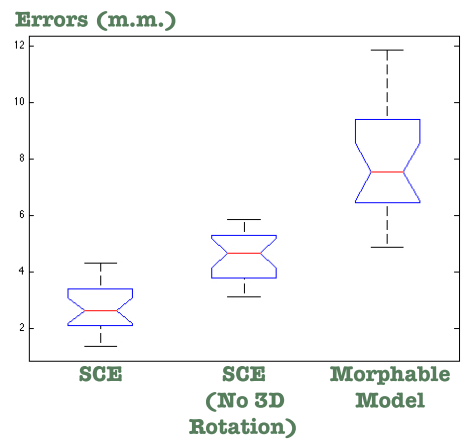

Fig. 3. ANOVA comparing our SCE, our SCE without 3D rotation matrices, and a 3D morphable model techniques in terms of their 3D errors to the ground truth models

\section{Evaluation}

We compare our conducted results with the AbS approach (a 3D morphable model in this case) in terms of visual appearance, precision, and speed. Because pure SfS is not suitable to create a complex shape of a human face, it excludes from our evaluation. The evaluation is conducted by firstly rendering thirty 3D ground truth face models with a Lambert shading to create their 2D shading images. Then, these shading images are used as inputs for a 3D reconstruction process. Figure 2 shows some visual results from the 3D reconstruction generated from a morphable model technique comparing to our Subspace Crossing Engine (SCE) and our SCE's initial 3D shape (without using a $3 \mathrm{D}$ rotation subspace). All visual result images are rendered from the reconstructed 3D faces with a Lambert shading. Figure 3 shows ANOVA of the 3D reconstruction errors (averaging from the whole face) of each approach by using Euclidean distance between vertices as a measurement from all thirty testing faces. In conclusion, our approach reconstructs $3 \mathrm{D}$ faces significantly closer to the ground truth faces than a morphable 
model. On average, the CPU time usages for our result is about 0.7 seconds $(0.4$ seconds without $3 \mathrm{D}$ rotation) comparing to about 74 seconds for a morphable model.

\section{Discussion and Future Work}

Our approach works well for a normal human face. However, if the input is non-human face such as a cartoon face, the base components will not be able to capture the shapes correctly and produce an unexpected result. Also, the input image must be in a controlled environment. To be precise, the input shading image must be strictly rendered with Lambert or similar shading model without texture. The input shading image must also be aligned (translating, scaling, and rotating) with the face template as much as possible. In the future, we plan to develop the system to be more flexible to these transformations, lighting condition, and shading model. The system will be very useful for passive low-cost 3D face capturing.

\section{References}

1. Zhang, R., Tsai, P.-S., Cryer, J.E., Shah, M.: Shape from shading: A survey. IEEE Transactions on Pattern Analysis and Machine Intelligence 21(8), 690-706 (1999)

2. Blanz, V., Vetter, T.: A morphable model for the synthesis of 3D faces. In: Proc. of SIGGRAPH 1999, pp. 187-194 (1999)

3. Kemelmacher-Shlizerman, I.: Internet-based morphable model. In: International Conference on Computer Vision, ICCV (2013)

4. Bourguignon, D., Chaine, R., Cani, M.-P., Drettakis, G.: Relief: A modeling by drawing tool. In: Proc. of Eurographics Workshop on Sketch-Based Interfaces and Modeling, pp. 151-160 (2004)

5. Gingold, Y., Igarashi, T., Zorin, D.: Structured annotations for 2D-to-3D modeling. ACM Transactions on Graphics (TOG) 28(5), 148 (2009)

6. DeCarlo, D., Metaxas, D., Stone, M.: An anthropometric face model using variational techniques. In: Proc. of SIGGRAPH 1998, pp. 67-74 (1998)

7. Blanz, V., Albrecht, I., Haber, J., Seidel, H.-P.: Creating face models from vague mental images. Comput. Graph. Forum 25(3), 645-654 (2006)

8. Blanz, V., Mehl, A., Vetter, T., Seidel, H.-P.: A statistical method for robust 3D surface reconstruction from sparse data. In: Proceedings of the 3D Data Processing, Visualization, and Transmission, 2nd International Symposium, 3DPVT 2004, pp. 293-300. IEEE Computer Society, Washington, DC (2004)

9. Kemelmacher, I., Basri, R.: 3D face reconstruction from a single image using a single reference face shape. IEEE Transactions on Pattern Analysis and Machine Intelligence 33, 394405 (2011)

10. Sumner, R.W., Popović, J.: Deformation transfer for triangle meshes. ACM Trans. Graph. 23(3), 399-405 (2004)

11. Sumner, R.W., Zwicker, M., Gotsman, C., Popović, J.: Mesh-based inverse kinematics. In: SIGGRAPH 2005: ACM SIGGRAPH 2005 Papers, pp. 488-495. ACM (2005)

12. Si, S., Tao, D., Geng, B.: Bregman divergence-based regularization for transfer subspace learning. IEEE Transactions on Knowledge and Data Engineering 22(7), 929-942 (2010) 